\title{
ReCONOCIMIENTO tAXONÓMICO DE DOS HOJAS Y UNA FLOR, incluidos en el ámbar del Mioceno de Chiapas, México
}

\author{
Carlos Castañeda-Posadas ${ }^{1 *}$, María de Jesús Hernández-Hernández², \\ Dulce María Figueroa-Castro', Rosa Emilia Pérez-Pérez ${ }^{1}$ \\ y Carlos Alberto Ruiz-Jiménez ${ }^{1}$ \\ ${ }^{1}$ Facultad de Ciencias Biológicas, Benemérita Universidad Autónoma de Puebla, Blvd. Valsequillo \\ y Av. San Claudio, Edificio 1 BIO 1 112-A, Ciudad Universitaria, Colonia Jardines de San Manuel. \\ Puebla, Puebla 72570, México. ${ }^{2}$ Posgrado en Ciencias Biológicas, Facultad de Ciencias Biológicas, \\ Benemérita Universidad Autónoma de Puebla. E-mail: *carlos.castaneda@correo.buap.mx
}

\begin{abstract}
Resumen
En el presente trabajo se muestran resultados de la identificación de cinco fósiles vegetales incluidos en ámbar de Simojovel, Chiapas. Las estructuras estudiadas corresponden a dos flores pertenecientes a la Familia Arecaceae; a dos hojas compuestas (foliolos) pertenecientes a la Familia Fabaceae y una hoja aislada perteneciente a la Familia Myrtaceae. La identificación y descripción de este tipo de estructuras fortalece el registro existente de la distribución de esas familias vegetales para el Mioceno en Chiapas. Este estudio contribuye a ampliar el conocimiento de la diversidad de restos vegetales incluidos en el ámbar de Chiapas.

Palabras Clave: Arecaceae, Fabaceae, Mioceno, Myrtaceae, fósil.
\end{abstract}

Taxonomic recognition of two leaves and one flower, included in the Miocene amber of Chiapas, Mexico

\begin{abstract}
In this study, the results of the identification of five plant fossils included in amber from Simojovel, Chiapas are shown. The vegetal structures studied correspond to two flowers which belong to the Arecaceae Family; two compound leaves (leaflets) which belong to the Fabaceae Family, and an isolated leaf, which belongs to the Myrtaceae Family. The identification and description of these kinds of structures strengthen the present records on the distribution of these family plants during the Miocene in Chiapas. This study contributes to widen the knowledge of the diversity of vegetal remains included in the amber from Chiapas.
\end{abstract}

Key Words: Areacaceae, Fabaceae, Miocene, Myrtaceae, fossil.

Nota: Artículo recibido el 13 de febrero del 2018 y aceptado el 08 de junio de 2018. 


\section{INTRODUCCIÓN}

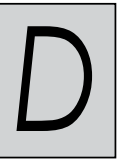

iversos estudios han documentado la existencia de restos vegetales fosilizados (Taylor, Taylor \& Krings, 2009). De estos, los fósiles vegetales mejor preservados son aquellos que se encuentran encapsulados en ámbar (i.e. resina vegetal fosilizada), ya que este tipo de fosilización permite una mejor visualización de estructuras delicadas en tercera dimensión (Avendaño-Gil, Carbot-Chanona \& Coutiño, 2012; Ytuarte-Núñez, 2014). Una gran variedad de inclusiones, tanto biológicas (microorganismos, restos vegetales y animales), como no biológicas (burbujas con contenidos gaseosos, líquidos y sedimento), (Avendaño-Gil, Carbot-Chanona \& Coutiño, 2012; Ytuarte-Núñez, 2014), se han encontrado preservadas en ámbar de depósitos de distintas edades alrededor del mundo (Poinar, 1998).

En México, los yacimientos de ámbar más importantes se localizan en el municipio de Simojovel de Allende, en el Estado de Chiapas. El ámbar de estos yacimientos se origina como resultado de la polimerización de la resina producida por las leguminosas Hymenaea mexicana (Poinar \& Brown, 2002) e Hymenaea allendis (Calvillo-Canadell, Cevallos-Ferriz \& RicoArce, 2010). Aunque numerosas inclusiones provenientes de estos yacimientos han sido estudiadas, el $73.64 \%$ corresponde a organismos animales y sólo el $26.36 \%$ a vegetales (Lowe, 2004; Ytuarte-Núñez, 2014). Esto deja de manifiesto que son pocos los trabajos paleobotánicos del ámbar de Chiapas (Ytuarte-Núñez, 2014).

El registro fósil de hojas, flores, semillas y frutos es muy importante, ya que a través de su estudio es posible conocer la diversidad de linajes presentes en distintas épocas (Taylor, Taylor \& Krings, 2009). Además, su estudio permite abundar en aspectos evolutivos, biogeográficos y ecológicos de los grupos vegetales existentes en el pasado, así como en la dinámica de los ecosistemas en los que se desarrollaron (Taylor, Taylor \& Krings, 2009). En el caso particular de los fósiles preservados en ámbar, es hasta las últimas décadas que se ha reconocido su importancia. A través del estudio de inclusiones vegetales en ámbar, es posible obtener mayor información y tratar de dar contestación a las preguntas ¿cómo era el ambiente de depósito?, ¿qué tipo de vegetación estaba establecida? y ¿qué tan parecida es la comunidad vegetal de esa época a la actual? Por ejemplo, a través del estudio de microfósiles (polen y esporas), de los estratos portadores de ámbar en Chiapas, se ha descrito a más de un centenar de géneros pertenecientes a 60 familias vegetales (Amaranthaceae, Aceraceae, Agavaceae, Anacardiaceae, Annonaceae, Apocynaceae, Aquifolaceae, Araliaceae, Arecaceae, Asteraceae, Betulaceae, Bignoniaceae, Bombacaceae, Boraginaceae, Bromeliaceae, Brunelliaceae, Burseraceae, Buxaceae, Capparaceae, Caprifoliaceae, Chloranthaceae, Clethraceae, Combretaceae, Cornaceae, Cyperaceae, Ericaceae, Euphorbiaceae, Fagaceae,
Gramineae, Hamamelidaceae, Hippocastanaceae, Juglandaceae, Lauraceae, Leguminosae, Liliaceae, Loranthaceae, Lythraceae, Malvaceae, Meliaceae, Moraceae, Myricaceae, Myrtaceae, Nyssaceae, Olacaceae, Onagraceae, Platanaceae, Polygalaceae, Rhizophoraceae, Rubiaceae, Rutaceae, Sabiaceae, Salicaceae, Sapindaceae, Sapotaceae, Smilacaceae, Styracaceae, Symplocaceae, Tiliaceae, Ulmaceae, y Urticaceae), así como de algunas Coniferales (Langenheim, Hackner \& Bartlett, 1967; Biaggi, 1978; Tomasini-Ortiz \& Martínez-Hernández, 1984; Martínez-Hernández, 1992; Palacios-Chávez \& Rzedowski, 1993; Graham, 1999).

Por su parte, los estudios con macrofósiles incluidos en ámbar miocénico de Simojovel han sido mucho más recientes. Por ello, el número de familias (Anacardiaceae, Arecaceae, Celastraceae, Euphorbiaceae, Fabaceae, Leguminosae, Malpighiaceae, Meliaceae, Rhamnaceae y Salicaceae) y especies [Acacia miranda (Miranda, 1963), Tapirira durhamii (Miranda, 1963), Hymenaea mexicana (Poinar \& Brown, 2002), Socratea brownii (Poinar, 2002), Swietenia miocenica (Castañeda-Posadas \& Cevallos-Ferriz, 2007), Hymenaea allendis (CalvilloCanadell, Cevallos-Ferriz \& Rico-Arce, 2010), Colpotrhinax chiapanensis (Chambers, Poinar \& Brown, 2012) y Lunania floresi (Hernández-Damián, Calvillo-Canadell \& CevallosFerriz, 2016)] vegetales reconocidas a la fecha, es mucho menor (Castañeda-Posadas \& Cevallos Ferriz, 2007; Calvillo-Canadell, Cevallos-Ferriz \& Rico-Arce, 2010; Chambers, Poinar \& Brown, 2012; Hernández-Damián, 2014; Hernández-Damián, Calvillo-Canadell \& Cevallos-Ferriz, 2016).

Por otra parte, la determinación del tipo de ambiente predominante durante el Mioceno en Chiapas, se ha realizado a través de análisis palinológicos. Los resultados de estos estudios sugieren la existencia de un clima cálido-húmedo, caracterizado por una vegetación de tipo selva tropical y manglar en las zonas de baja elevación y de bosque mesófilo o bosque templado en las zonas de mayor altitud (Langenheim, Hackner \& Bartlett, 1967; Biaggi, 1978; Martínez-Hernández, 1992; Palacios-Chávez \& Rzedowski, 1993; Graham, 1999; Castañeda-Posadas \& Cevallos-Ferriz, 2007). De manera similar, las inclusiones de macrofósiles vegetales que se han estudiado sustentan cada vez más esas hipótesis y refinan las reconstrucciones ambientales. Por ello y porque existe una notable carencia de estudios con macrofósiles de la localidad (i.e. los estudios actuales no representan ni el 10\% de las familias que se han descrito con microfósiles), en este trabajo se reportan nuevos registros de macrofósiles miocénicos de familias vegetales del Estado de Chiapas, contribuyendo así a robustecer el acervo de grupos biológicos registrados para la región.

\section{Área de estudio}

El presente estudio se realizó con inclusiones de ámbar provenientes de Simojovel de Allende, en el Estado de Chiapas 


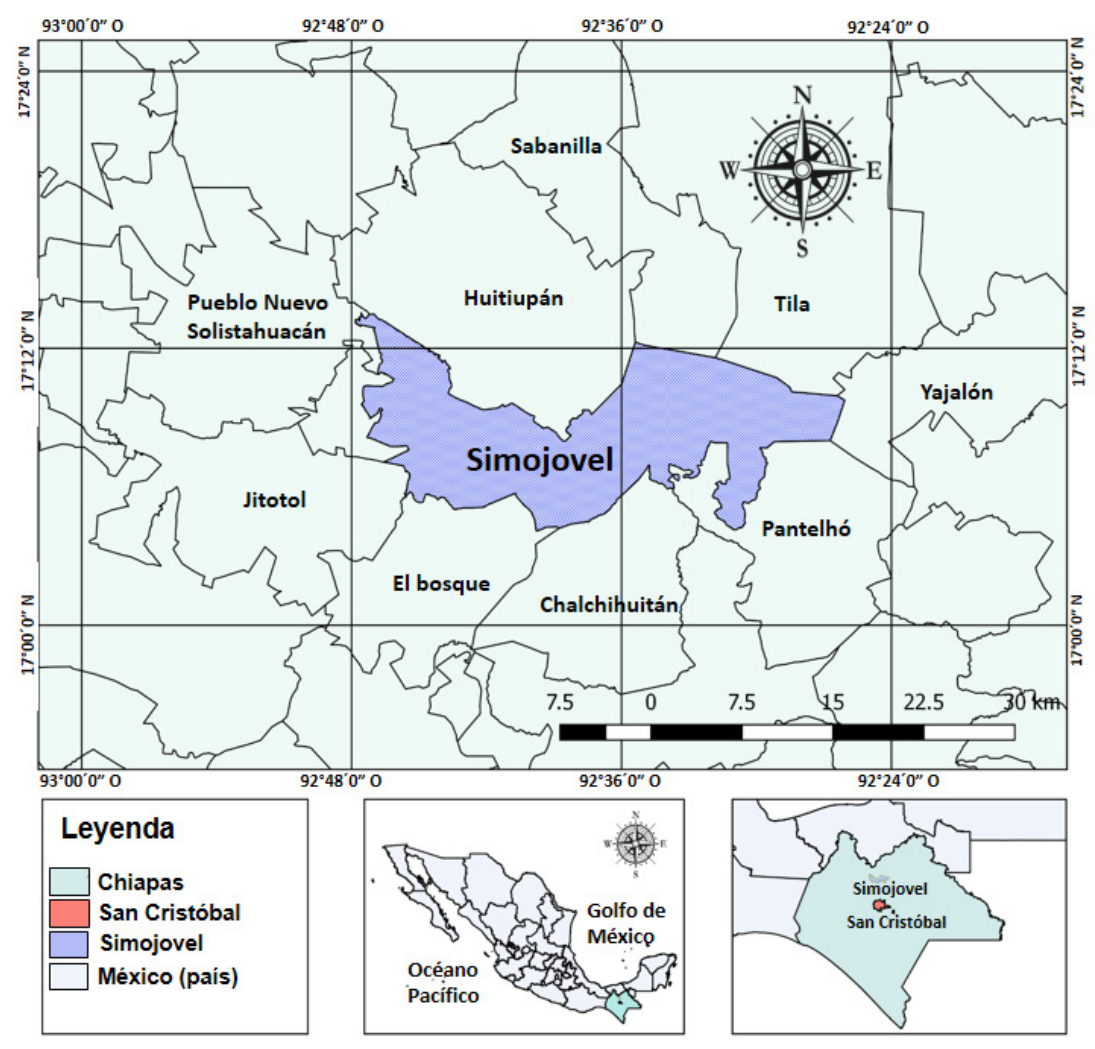

Figura 1. Área de Estudio, mostrando la ubicación de la Localidad de Simojovel dentro del Estado de Chiapas. Ver la versión a color en línea.

$\left(17^{\circ} 08^{\prime} 19^{\prime \prime} \mathrm{N}\right.$ y $92^{\circ} 43^{\prime} 00^{\prime \prime} \mathrm{W}$, a una altitud de $600 \mathrm{msnm}$; Figura 1). Las inclusiones de ámbar proceden de estratos de lutita carbonosa intercalados con los de las Formaciones Mazantic (esquisto) y Balumtum (arenisca) y tienen una edad estimada para el Mioceno Temprano-Medio, determinada a partir de la presencia de moluscos de los géneros Turbinella Lamarck, 1799 y Melongena Schumacher, 1817, incluidos en el ámbar (Vega, Nyborg, Coutiño, Solé \& Hernández-Monzón, 2009; Perrilliat, Vega \& Coutiño, 2010). Los depósitos de Simojovel de Allende están asociados a un ambiente de línea de costa y a tierras bajas continentales (Perrilliat, Vega \& Coutiño, 2010; Riquelme et al., 2014).

\section{MAteriales Y mÉtOdos}

El material estudiado consta de dos fósiles vegetales incluidos en una pieza de ámbar, pertenecientes a la Colección Nacional de Paleontología "María del Carmen Perrilliat" de la Universidad Nacional Autónoma de México; un fósil incluido en una pieza, perteneciente a la Colección del Museo "Lilia Mijangos" y dos fósiles incluidos en una pieza, provenientes de la Colección de Paleontología de la Benemérita Universidad Autónoma de Puebla.
Para determinar la afinidad de los organismos preservados en los fragmentos de ámbar, los macrofósiles fueron observados y fotografiados con un microscopio estereoscópico marca MOTIC modelo SM2168TL equipado con una cámara modelo MOTICam 2000 de 3 megapixeles con puerto USB. Las imágenes obtenidas se procesaron con el programa Motic images plus 2.0. La afinidad de los macrofósiles se llevó a cabo empleando las bases de datos Delta (http://delta-intkey.com/angio/ident.htm) y Famex (http://www.abatax.abacoac.org/clavesTax_lista.php), bibliografía especializada (Cronquist, 1981; Takhtajan, 2009; Simpson, 2010) y revisiones de ejemplares herborizados en el herbario virtual del Consejo Nacional para la Biodiversidad (CONABIO) y el Herbario Nacional de México (MEXU).

\section{Resultados}

\section{Flor fósil IGMPB 1353 e IGMPB 1354}

Holotipo: IGMPB 1353, Colección Nacional de Paleontología "María del Carmen Perrilliat", Instituto de Geología, UNAM, México.

Paratipo: IGMPB 1354, Colección Nacional de Paleontología "María del Carmen Perrilliat", Instituto de Geología, UNAM, México. 

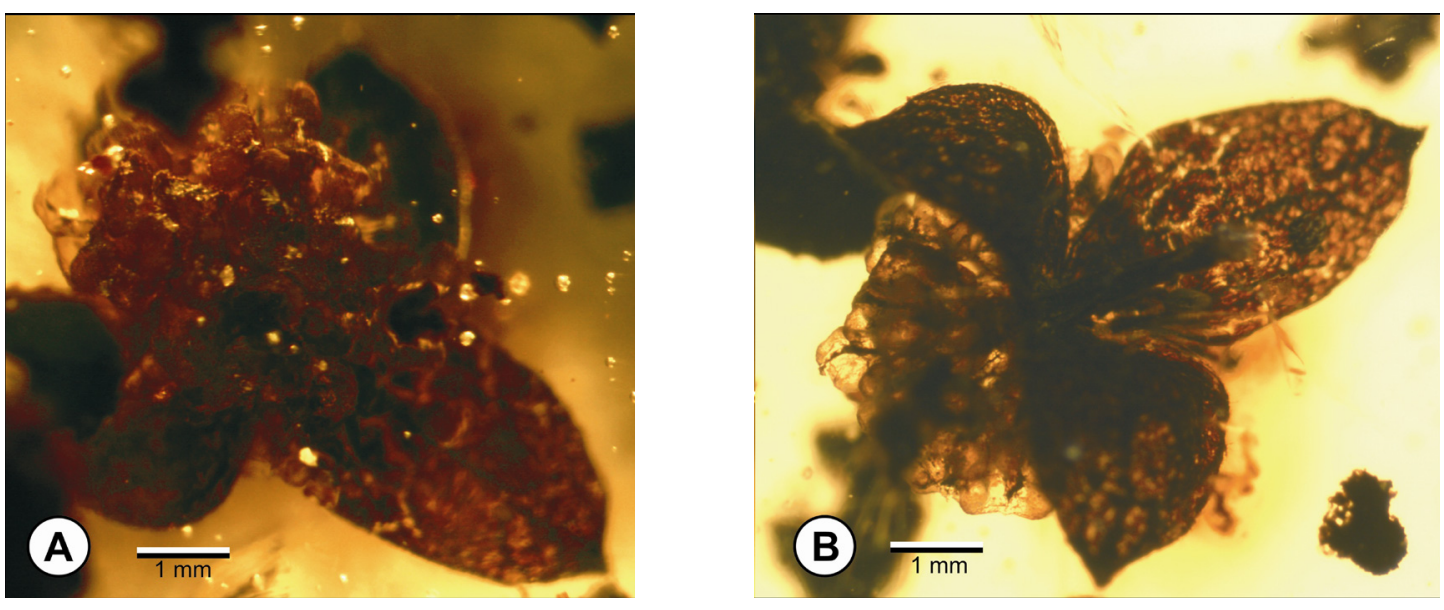

Figura 2. Fósil IGMPB 1353. A) Flor en vista superior, se observa la disposición de los estambres. B) Flor, se observa el pedicelo y el perianto compuesto por tres tépalos. Escala= $1 \mathrm{~mm}$ (Fotos tomadas por María de Jesús Hernández Hernández). Ver la versión a color en línea.

Descripción: Se analizaron dos estructuras florales completas incluidas en una pieza de ámbar de $3 \mathrm{~cm}$ de largo por 1.5 $\mathrm{cm}$ de ancho. La flor es de talla pequeña $(3.49 \mathrm{~mm}$ de largo por $2.16 \mathrm{~mm}$ de ancho), con pedicelo de $1.96 \mathrm{~mm}$ de largo y pequeñas espinas. La flor tiene simetría actinomórfica y es trímera. El perianto está compuesto por tres tépalos libres de $1.55 \mathrm{~mm}$ de largo, glabros. Se considera que la flor es masculina (estaminada), por la presencia de numerosos estambres globosos, posiblemente más de 30; la antera es lobulada o globosa, con dehiscencia longitudinal. No se logró observar un gineceo o su remanente, por lo que se infiere que es una flor unisexual masculina (Figura 2).

Afinidad botánica: caracteres como el tamaño pequeño de la flor, la simetría actinomorfa, flor unisexual (estaminada), perianto conformado por tépalos y antera basifija con dehiscencia longitudinal, sugieren que el fósil tiene afinidad con las familias Juncaceae y Arecaceae. Sin embargo, un análisis más detallado de los caracteres morfológicos de la Familia Juncaceae indica que sus flores se caracterizan por la presencia de un perianto de tipo glumáceo (con glumas) y pocos estambres (3-6 solamente), por lo que se descarta la afinidad de los macrofósiles con esta familia. Por otro lado, las flores de la Familia Arecaceae tienen de 3 a más de 200 estambres, son trímeras, en algunos casos presentan un perianto compuesto por tépalos y pueden ser pediceladas o sésiles. Estos caracteres son afines con los del macrofósil estudiado, por lo que se asignó a dicha Familia (Quero, 1994, 2004; Dransfield \& Uhl , 1998).

\section{Foliolo fósil BUAPAL 236 y 237}

Holotipo: BUAPAL 236 Colección de Paleontología de la Benemérita Universidad Autónoma de Puebla.

Paratipo: BUAPAL 237 Colección de Paleontología de la Benemérita Universidad Autónoma de Puebla
Descripción: Se analizaron dos foliolos incluidos en una pieza de ámbar de $3.5 \mathrm{~cm}$ de ancho por $8.5 \mathrm{~cm}$ de largo. Los macrofósiles corresponden a hojas compuestas, bipinnadas, constituidas por 10 a 34 pares de foliolulos insertados de manera opuesta. Los foliolos tienen un largo de $17.5 \mathrm{~mm}$ y un ancho de $6 \mathrm{~mm}$. Los foliolulos son asimétricos, sésiles, con margen entero, forma ovada-ovobada, base truncada y ápice agudo a acuminado. La lámina es glabra a pubérula. La venación media es excéntrica, de tipo broquidódroma (Figura 3).

Afinidad Botánica. Los caracteres hojas compuestas, bipinnadas, tamaño pequeño del foliolo, márgenes enteros y vena media excéntrica tipo broquidódroma, están presentes en pocos grupos de plantas, pero son muy comunes entre las leguminosas (Grether, 2001; Martínez-Bernal, Grether \& González-Amaro, 2008). En particular, la Subfamilia Mimosoideae se caracteriza por tener hojas alternas con filotaxia espiralada, hojas pinnadas o bipinnadas, de una a numerosas pinnas dispuestas en pares, de uno a varios pares de foliolos o reducidos a filodios, pulvínulos presentes y estípulas generalmente pequeñas (Martínez-Bernal, Grether \& González-Amaro, 2008).

\section{Hoja fósil LZ.MALM74}

Holotipo: LZ.MALM74. Colección del museo de Ámbar "Lilia Mijangos".

Descripción: La pieza de ámbar analizada tiene $8.21 \mathrm{~cm}$ de longitud por $4.5 \mathrm{~cm}$ de ancho y tiene incluidos distintos restos de animales de la clase Insecta, así como estructuras foliares. La hoja presenta una textura coriácea (Figura 4A), tiene una longitud de $1.9 \mathrm{~cm}$ y presenta peciolo. La lámina es elíptica y simétrica, con margen entero, de tamaño nanófilo, con ápice y base convexos. La venación de primer orden es de tipo pinnada, con una vena media recta, ligeramente más gruesa hacia la base. La venación de segundo orden es de tipo broquidódromo, con 


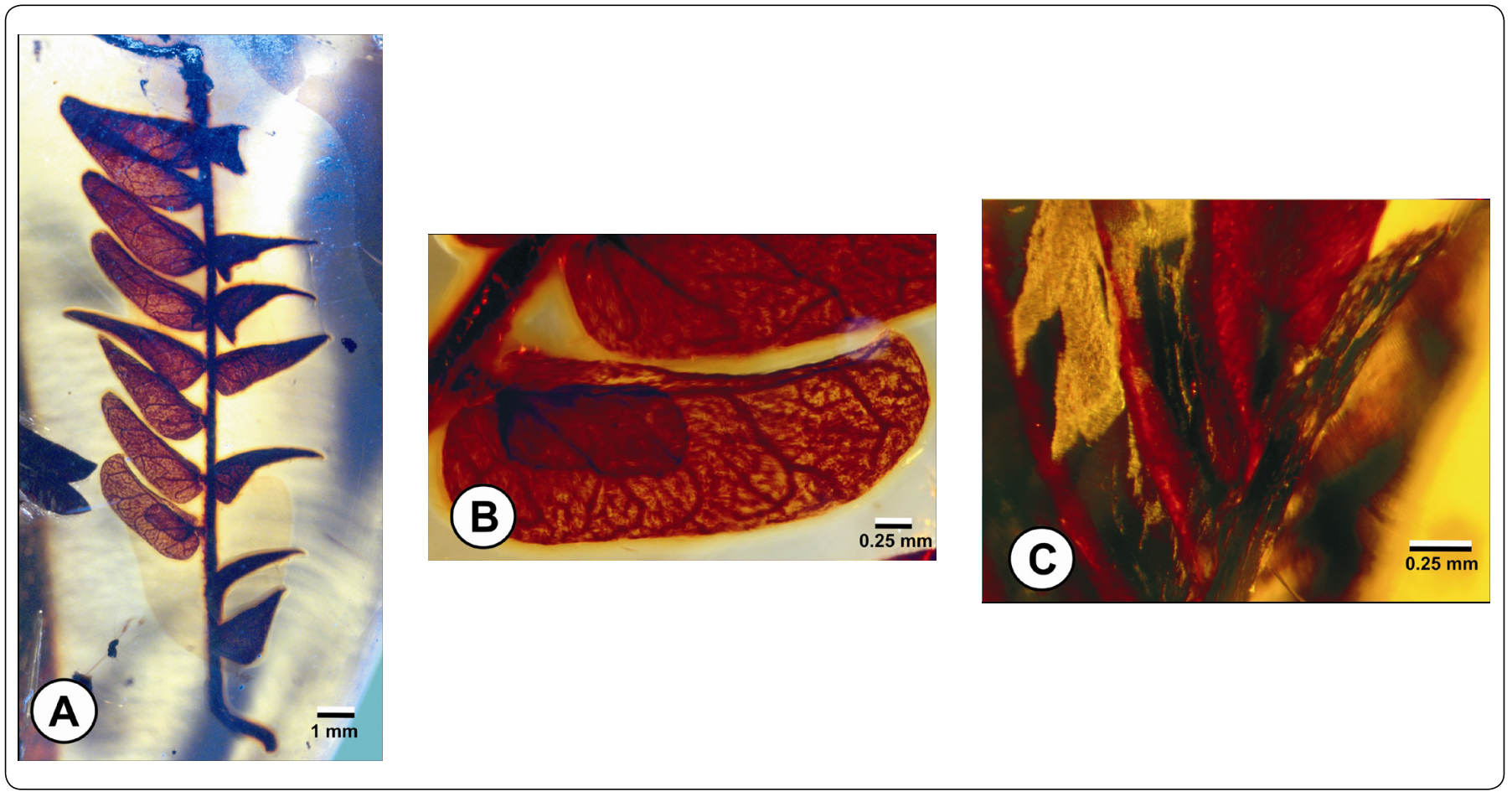

Figura 3. Fósil BUAPAL 236. A) Foliolo bipinnado, compuesto por foliolulos opuestos. B) Foliolulo con venación excéntrica broquidódroma. C) Tallo mostrando el pulvínulo. (Fotos tomadas por Carlos Castañeda-Posadas). Ver la versión a color en línea.

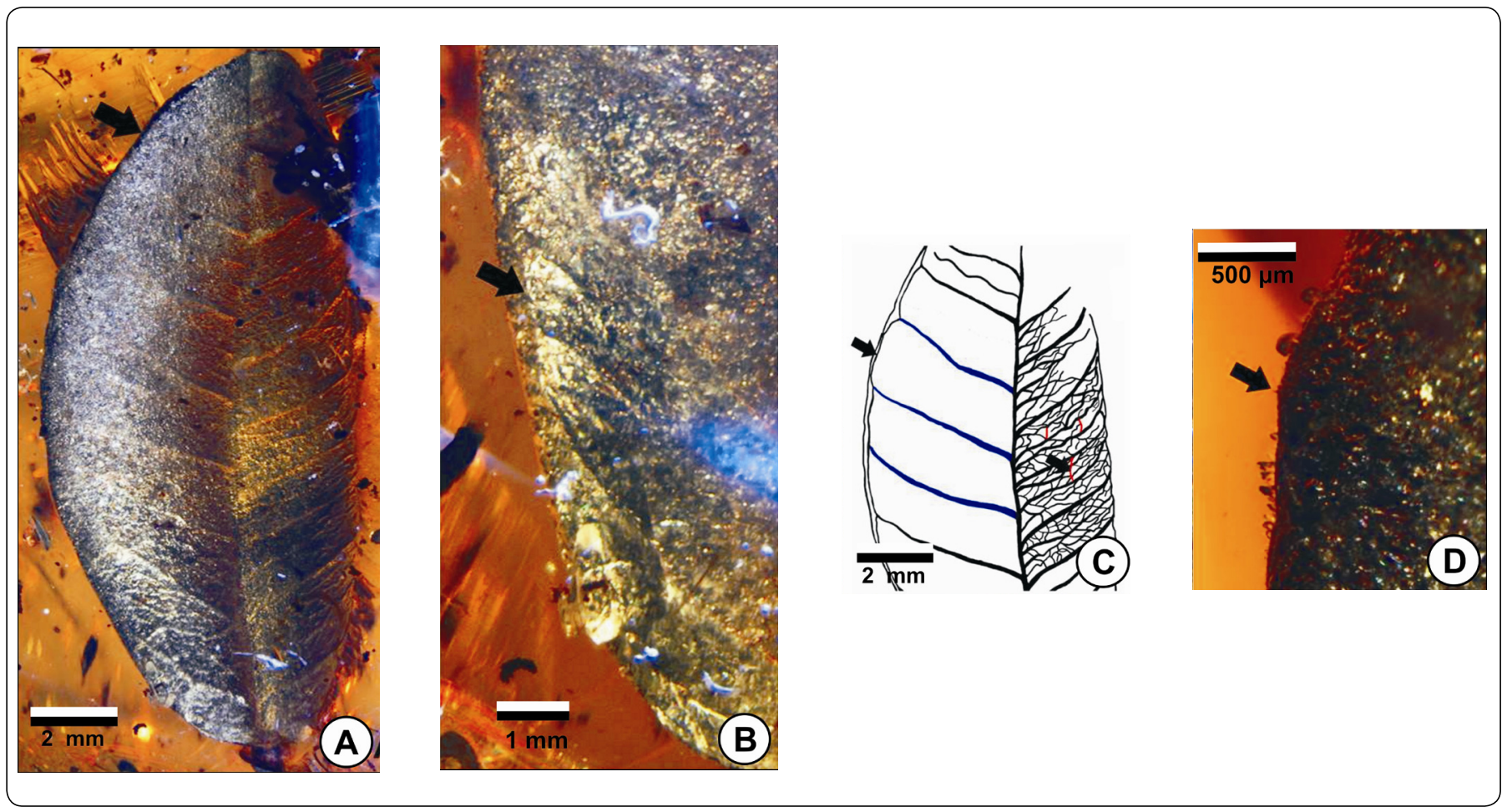

Figura 4. Fósil LZ.MALM74. A) Se muestra la hoja elíptica, la venación de primer orden de tipo pinnada (flecha), las venas de segundo orden de tipo broquidódromo y el margen entero (flecha izquierda); B) vena intramarginal. Escala= $2 \mathrm{~mm}$. C) Esquema de la hoja con los órdenes de venación, resaltando la posición paralela de las venas de tercer orden en relación a la vena media. D) Tricomas glandulares. Ver la versión a color en línea. 
presencia de una vena intramarginal. La venación de tercer orden es reticulada al azar, con un curso de venas muy ramificadas. La hoja presenta tricomas visibles, simples, alargados, cilíndricos y de tipo no glandular.

Afinidad taxonómica. Los caracteres morfológicos hojas simples, margen entero, venación de primer orden tipo pinnada y de segundo orden tipo broquidódromo y tricomas cilíndricos en el margen, sugieren una afinidad con las familias Annonaceae, Monimiaceae, Clusiaceae, Myrsinaceae, Fabaceae, Thymelaeaceae, Myrtaceae, Burseraceae, Rutaceae, Acanthaceae y Convolvulaceae. Al realizar una revisión más exhaustiva de los caracteres que presenta el macrofósil de Simojovel y comparándolo con los ejemplares de herbarios, se descarta a las familias Burseraceae, Monimiaceae, Clusiaceae, Myrsinaceae, Rutaceae, Thymelaeaceae y Acanthaceae, ya que estos grupos no presentan venación reticulada de tercer y cuarto orden; en estos grupos la venación es tipo percurrente opuesta o alterna. La Familia Fabaceae se descarta porque la mayoría de las especies presentan una venación primaria tipo palmada y aunque existen especies que tienen venación tipo pinnada, al revisarlas se observa que los ángulos de inclinación son distintos a los observados en el macrofósil. Además, en la Familia Fabaceae el arreglo de las venas de tercer y cuarto orden regularmente es percurrente, mientras que en el macrofósil el arreglo es de tipo reticulado al azar. Por otro lado, las características del macrofósil ya mencionadas además de la presencia de una vena intramarginal, venas de segunda categoría con disposición paralela y venas de tercer categoría a veces paralelas a la vena media, se encuentra una fuerte afinidad con los rasgos distintivos de la Familia Myrtaceae, la cual tiene un patrón de venación muy consistente (Manchester, Dilcher \& Wing, 1998; González, 2011a, 2011b; Wilson, 2011).

\section{Discusión}

Los caracteres anatómicos y morfológicos de las plantas nos ayudan a obtener su identificación taxonómica a diferentes niveles. En este trabajo se muestra la descripción y compatibilidad taxonómica a nivel familia de cinco estructuras vegetales incluidas en ámbar de Simojovel, Chiapas. Se reconoció a la Familia Arecaceae por dos estructuras florales, a la Familia Fabaceae por dos estructuras vegetativas (foliolos) y a la Familia Myrtaceae por una estructura foliar.

El registro fósil de la familia Arecaceae es muy amplio y diverso; incluyendo maderas, hojas, inflorescencias, flores, frutos y polen (Harley, 2006). El reporte más antiguo es para el Cretácico, proveniente de una localidad en Egipto (Harley, 2006), mientras que para México se tiene representada en un afloramiento con una edad del Cretácico Superior(Sainz-Reséndiz, Estrada-Ruiz, Mateo-Cid \& Porras-Múzquiz, 2015). El registro fósil de esta familia preservado en ámbar comprende dos nuevas especies de República Dominicana descritas por Poinar (2002), una del género Trithrinax y otra del género Socratea y una nueva especie del género Colpothrinax descrita por Chambers, Poinar \& Brown (2012), para una localidad en México. De acuerdo con lo anterior, los macrofósiles incluidos en ámbar identificados en el presente estudio como pertenecientes a la Familia Arecaceae, son el segundo reporte para el territorio nacional y el tercero a nivel internacional.

La Familia Myrtaceae ha sido reportada a partir del estudio de macrofósiles en distintas partes del mundo. Por ejemplo, ha sido documentada para el periodo Cretácico tardío en la Antártida (Poole, Hunt \& Cantrill, 2001; Poole, Mennega \& Cantrill, 2003) y para el Eoceno en Colorado, Estados Unidos (Manchester, Dilcher \& Wing, 1998), Argentina (Wilf et al., 2005), Chile (Gayo, Hinojosa \& Villagrán, 2005), Australia y Nueva Zelanda (Wilson, 2011). En México, los únicos representantes fósiles de la Familia Myrtaceae provienen de la localidad miocénica de Ixtapa, Chiapas (Peralta, 2009). Por lo tanto, el macrofósil identificado en el presente estudio constituye el segundo reporte de la familia para el Mioceno, tanto para Chiapas como para México. Además, es el primer macrofósil de la Familia Myrtaceae incluido en ámbar a nivel mundial.

Por último, el registro de la Familia Fabaceae (Leguminosae) es muy diverso, en cuanto a estructuras fosilizadas descritas (maderas, hojas, flores, frutos y semillas). Los fósiles más antiguos de esta familia provienen del Cretácico (Herendeen, Crepet \& Dilcher, 1992). En México, la Familia Fabaceae data del Oligoceno y proviene del Estado de Puebla (CalvilloCanadell \& Cevallos-Ferriz, 2005). Los foliolulos identificados en el presente estudio amplían la distribución de la familia en el país y son el primer reporte de estas estructuras en el ámbar de Chiapas.

\section{Conclusiones}

La descripción e identificación de los macrofósiles analizados en el presente estudio permite concluir que el fósil BUAPAL 236 adjudicado a la Familia Myrtaceae constituye, al menos temporalmente, el registro más sureño de la familia para el Mioceno, ya que no se contaba con registros de macrofósiles de esa familia. Los macrofósiles IGMPB 1353 e IGMPB 1354 y LZ.MALM74 correspondientes a las familias Arecaceae y Fabaceae, respectivamente, coinciden con reportes previos obtenidos a partir de análisis palinológicos y flores incluidas en ámbar. Las tres familias vegetales a las que corresponden los macrofósiles descritos en este estudio, presentan actualmente una distribución tropical y subtropical, lo que refuerza la hipótesis planteada por Graham (1999), acerca de que la vegetación de Simojovel de Allende durante el Mioceno Temprano-Medio se asemejaba a un bosque tropical-subtropical. Sin embargo, aún existen muchas macro-estructuras vegetales por estudiar. Es importante continuar con este tipo de estudios, no sólo por que proporcionan información valiosa sobre los ambientes 
del pasado, sino también porque los registros paleontológicos preservados en ámbar están en riesgo de perderse debido a su intensa comercialización.

\section{AgRAdeCIMIENTOS}

Los autores agradecen a los Responsables tanto de la Colección Nacional de Paleontología "María del Carmen Perrilliat" como del Museo de ámbar "Lilia Mijangos", por el préstamo del material. El estudio fue financiado por la VIEP-BUAP (CAPCNAT15-1) a CCP.

\section{Referencias}

Avendaño-Gil, J., Carbot-Chanona, G. \& Coutiño, J.M.A. (2012). Estudio del ámbar con inclusiones biológicas de la Colección Paleontológica de la Secretaría de Medio Ambiente e Historia Natural, Chiapas, México. Lacandonia: Revista de Ciencias UNICACH, 6, 23-29. http://cuid.unicach.mx/revistas/index. php/lacandonia/article/view/27.

Biaggi, R.E.(1978). Palynology and paleoecology of someOligo-Miocene sediments from Chiapas, México. Walla College, California, EEUU. Tesis de Maestría, Universidad California. 64 pp.

Calvillo-Canadell, L. \& Cevallos-Ferriz, S.R.S. (2005). Diverse assemblage of Eocene and Oligocene Leguminosae from Mexico. International Journal of Plant Science, 166, 671-692.

Calvillo-Canadell, L., Cevallos-Ferriz, S.R.S. \& Rico-Arce, L. (2010). Miocene Hymenaea flowers preserved in amber from Simojovel de Allende, Chiapas, Mexico. Review of Palaeobotany and Palynology, 160, 126-134. https://doi.org/10.1086/430096.

Castañeda-Posadas, C. \& Cevallos-Ferriz, S.R.S. (2007). Swietenia (Meliaceae) flower in Late Oligocene-Early Miocene Amber from Simojovel de Allende, Chiapas, Mexico. American Journal of Botany, 4, 1821-1827. DOI: 10.3732/ajb.94.11.1821.

Chambers, K.L., Poinar Jr., G.O. \& Brown, A.E. (2012). A new fossil species of Colpothrinax (Arecaceae) from Mid-Tertiary Mexican amber. Journal of the Botanical Research Institute of Texas, 6, 557-560. http://www.jstor.org/stable/41972439.

Cronquist, A. (1981). An Integrated System of Classification of Flowering Plants. Columbia University Press, Nueva York. $1262 \mathrm{pp}$.

Dransfield, J. \& Uhl, N.W.(1998). Palmae. Pp. 306-389. En: Kubitzki, K. (ed.). The families and genera of vascular plants. IV. Flowering Plants Monocotyledons: Alismatanae and Commelinanae. Springer-Verlag, Berlin.

Gayo, E., Hinojosa, L.F. \& Villagrán, C. (2005). On the persistence of tropical paleofloras in central Chile during the early Eocene. Review of Paleobotany and Palynology, 137, 41-50. https:// doi.org/10.1016/j.revpalbo.2005.09.001.

González, C. (2011a). Arquitectura foliar de las especies de Myrtaceae nativas de la Argentina I: grupos "Myrcia", "Myrceugenia" y "Plinia". Boletín de la Sociedad Argentina de Botánica, 46, 41-63.

González, C. (2011b). Arquitectura foliar de las especies de Myrtaceae nativas de la flora Argentina III: Grupo "Eugenia". Boletín de la Sociedad Argentina de Botánica, 46, 85 - 104.

Graham, A. (1999). Studies in Neotropical paleobotany. XIII. An Oligo-Miocene palynoflora from Simojovel(Chiapas, Mexico). American Journal of Botany, 86, 17-31. DOI: 10.2307/2656951.

Grether, R. (2001). Mimosa. En: Flora de Nicaragua: Systematic Botany Monographs, 85 (2), 1487-1497.

Harley, M.M. (2006). A summary of fossil record for Arecaceae. Botanical Journal of the Linnean Society, 151, 39-67. DOI: 10.1111/j.1095-8339.2006.00522.x.

Herendeen, P.S., Crepet, W.L. \& Dilcher, D.L. (1992). The fossil history of the Leguminosae: phylogenetic and biogeographic implications. Pp. 303-316. En: Herendeen, P.S., Dilcher, D.L. (eds.). Advances in legume systematics. Pt. 4. The fossil record. Royal Botanical Gardens, Kew.

Hernández-Damián, A.L. (2014). Flores preservadas en ámbar de Simojovel, Allende, Chiapas, México. Tesis de Maestría, Universidad Nacional Autónoma de México, México. 176 págs.

Hernández-Damián, A.L., Calvillo-Canadell, L. \& Cevallos-Ferriz, S.R.S. (2016). Flor de una nueva especie de Lunania Hook. (Salicaceae sensu lato-Samydeae) incluida en ámbar del Mioceno de Simojovel de Allende, Chiapas, México. Boletín de la Sociedad Geológica Mexicana, 68, 29-36.

Langenheim, J.H., Hackner, B.L. \& Bartlett, A. (1967). Mangrove pollen at the depositional site of Oligo-Miocene amber from Chiapas, Mexico. Botanical Museum Leaflets, Harvard University, 21, 289-324.

Lowe, L.S. (2004). El ámbar de Chiapas y su distribución en Mesoamérica. Instituto de Investigaciones Filológicas, Centro de Estudios Mayas, UNAM, México.171 págs.

Manchester, S.R., Dilcher, D.L. \& Wing, S.L. (1998). Attached leaves and fruits of myrtaceous affinity from the middle Eocene of Colorado. Review of Palaeobotany and Palynology, 102, 153- 163 .

Martínez-Hernández, E. (1992). Caracterización ambiental del Terciario de la región de Ixtapa, Estado de Chiapas: un enfoque palinoestratigráfico. Revista del Instituto de Geología, UNAM, 10, 54-64.

Martínez-Bernal, A., Grether, R. \& González Amaro, R.M. (2008). Flora de Veracruz: 1, Mimosoidae: Mimosa. Leguminosae. Fascículo 147. Instituto de Ecologia, A.C., Xalapa, Veracruz, México, 127 págs.

Miranda, F. (1963). Two plants from the amber of the Simojovel, Chiapas, Mexico, area. Journal of Paleontology, 36, 611-614.

Palacios-Chávez, R. \& Rzedowski, J. (1993). Estudio palinológico de las floras fósiles del Mioceno inferior y principios del Mioceno medio de la región de Pichucalco, Chiapas, México. Acta Botánica Mexicana, 24, 1-96.

Peralta, E. (2009). Arquitectura Foliar de hojas fósiles de Ixtapa, Chiapas, México. Tesis de Licenciatura, Universidad Nacional Autónoma de México, México. 77 págs.

Perrilliat, M.C., Vega, F.J. \& Coutiño, M.A. (2010). Miocene mollusks from the Simojovel area in Chiapas, southwestern Mexico. Journal of South American Earth Sciences, 30, 111-119. https:// doi.org/10.1016/j.jsames.2010.04.005. 
Poinar Jr., G.O. (1998). Fossils explained: Palaeontology of amber. Geology Today, 14, 154-160.

Poinar Jr., G. (2002). Fossil palm flowers in Dominican and Mexican amber. Botanical Journal of the Linnean Society, 138, 57-61. DOI: 10.1046/j.1095-8339.2002.00010.x.

Poinar Jr., G. \& Brown, A.E. (2002). Hymenaea mexicana sp. nov. (Leguminosae: Caesalpinioideae) from Mexican amber indicates Old World connections. Botanical Journal of the Linnean Society, 139, 125-132. DOI: 10.1046/j.10958339.2002.00053.x.

Poole, I., Hunt, R.J. \& Cantrill, D.J. (2001). A fossil wood flora from King George Island: ecological implications for an Antarctic Eocene vegetation. Annals of Botany, 88, 33-54. https://doi. org/10.1006/anbo.2001.1425.

Poole, I., Mennega, A.M.W. \& Cantrill, D.J. (2003). Valdivian ecosystems in the Late Cretaceous and Early Tertiary of Antarctica: further evidence from myrtaceous and eucryphiaceous fossil wood. Review of Palaeobotany and Palynology, 124, 9-27. https://doi.org/10.1016/S00346667(02)00244-0.

Quero, H.J. (1994). Flora de Veracruz: Palmae. Instituto de Ecología, A.C., Xalapa, Veracruz, México, 40 págs.

Quero, H.J. (2004). Flora del Bajío y de Regiones Adyacentes: Palmae. Fascículo 129. Instituto de Ecología, A.C., Xalapa, Veracruz, México, 29 págs.

Riquelme, F., Northrup, P., Ruvalcaba-Sil, J.L., Stojanoff, V., Siddons, D.P. \& Alvarado-Ortega, J. (2014). Insights into molecular chemistry of Chiapas amber using infrared-light microscopy, PIXE/RBS, and sulfur K-edge XANES spectroscopy. Applied Physics, A116,
97-109. https://doi.org/10.1007/s00339-013-8185-2.

Sainz-Reséndiz, A., Estrada-Ruiz, E., Mateo-Cid, L.M. \& PorrasMúzquiz, H. (2015). Primer registro de un estípite de Coryphoideae: Palmoxylon kikaapoa de la Formación Olmos del Cretácico Superior, Coahuila, México. Revista Mexicana de Biodiversidad 86, 872-881. https://doi.org/10.1016/j. rmb.2015.09.009.

Simpson, M.G. (2010). Plant Systematics. Academic Press, China. Takhtajan, A. (2009). Flowering Plants. Springer, Netherlands.

Taylor, T.N., Taylor, E.L. \& Krings, M. (2009). Paleobotany. The Biology and Evolution of Fossil Plants. Academic Press, USA. 1230 págs.

Tomasini-Ortiz, A.C. \& Martínez-Hernández, E. (1984). Palinología del Eoceno Oligoceno de Simojovel, Chiapas. Paleontología Mexicana, 50, 1- 61 .

Vega, F.J., Nyborg, T., Coutiño, M.A., Solé, J. \& Hernández-Monzón, O. (2009). Neogene Crustacea from southeastern Mexico. Bulletin of the Mizunami Fossil Museum, 35, 51-69. http:// dx.doi.org/10.18268/BSGM2017v69n3a8.

Wilf, P., Johnson, K.R., Cúneo, N.R., Smith, M.E., Singer, B.S. \& Gandolfo, M.A. (2005). Eocene plant diversity at Laguna del Hunco and Río Pichileufú, Patagonia, Argentina. American Naturalist, 165, 634-650. https://doi.org/10.1086/430055.

Wilson, G.P. (2011). Myrtaceae. Pp. 212-271. En: Kubizki, K. (ed.). The families and genera of vascular plants. Volume X. Springer, Heidelberg, Alemania.

Ytuarte- Núñez, C. (2014). Cultura y comunicación en el intercambio global de mercancías: el ámbar de Chiapas, México. Universidad Autónoma de Chiapas, Chiapas. 219 págs. 